\title{
A MISDIAGNOSED CASE OF HANSEN'S DISEASE
}

\author{
Pranit Vijaykumar Farande ${ }^{1}$, Amita Mhatre ${ }^{2}$, Sunil Petkar ${ }^{3}$, Rikita Patel ${ }^{4}$
}

${ }^{1}$ Assistant Professor, MBBS, DNB, DVD, Department of Dermatology and Venereology, Terna Medical College, Nerul.

${ }_{2}^{2}$ Resident, MBBS, DDV, Department of Dermatology and Venereology, Terna Medical College, Nerul.

${ }^{3}$ Professor, MBBS, MD, Department of Dermatology and Venereology, Terna Medical College, Nerul.

${ }^{4}$ Resident, MBBS, Department of Dermatology and Venereology, Terna Medical College, Nerul.

HOW TO CITE THIS ARTICLE: Farande PV, Mhatre A, Petkar S, et al. A misdiagnosed case of Hansen's disease. J. Evolution Med. Dent. Sci. 2017;6(80):5676-5677, DOI: 10.14260/jemds/2017/1231

\section{PRESENTATION OF CASE}

A 24 years male patient hailing from Bhagalpur, Bihar, presently residing at Kopar Khairane, Navi Mumbai came with chief complaints of multiple, round, elevated skin lesions on face and multiple light coloured skin lesions on neck and back since 1 year.

Patient was apparently alright one year back when he developed multiple, light coloured skin lesions on face, neck and back. The lesions were initially of pea size, then gradually increased in size over a period of 1 year. Six months later he developed multiple, round, brownish red, elevated lesions on the face. Patient took medical advice at his native place in Bihar. Screening test for Post Kala-Azar Dermal Leishmaniasis (PKDL)- K39 was done, which was negative. $\mathrm{He}$ was investigated further and on clinico-pathological correlation started on MBMDT one and a half months back with no improvement.

There was no history of bleeding from nose, loss of hair, decreased sweating or decreased sensations over affected areas. There was no history of muscle weakness or any deformity. He did not have any history of fever, weight loss, tingling or numbness. There was no history of similar complaints in the past. Family history and personal history were not significant. Patient gave history of tobacco chewing since 10 years.

On examination, his vitals were stable. There was no pallor, icterus, cyanosis, clubbing, lymphadenopathy or oedema.

On cutaneous examination there were multiple, brownish red, infiltrating papules and nodules distributed over the face, smallest being $0.5 \mathrm{~cm}$ and largest being $2 \mathrm{~cm}$. There were nodules over forehead just above the eyebrows coalescing to form plaques. But there was no ciliary or superciliary hair loss. There were multiple, reddish-yellow xanthomatous papules over chin. Two papules of approximately $0.5 \mathrm{~cm}$ were seen over right ear, but left ear had no lesions. There was no infiltration of ear lobe. There were multiple, well defined, irregular hypopigmented macules and patches seen distributed all over the back, smallest being $0.5 \mathrm{~cm}$ and largest being $3 \mathrm{~cm}$. The sensations were intact. Peripheral

'Financial or Other Competing Interest': None.

Submission 16-09-2017, Peer Review 28-09-2017,

Acceptance 30-09-2017, Published 05-10-2017.

Corresponding Author:

Dr. Pranit Vijaykumar Farande,

Flat-3, Nishant Kutir CHS,

Plot-216, Sector-28, Vashi,

Navi Mumbai-400703.

E-mail:fpranit@gmail.com

DOI: $10.14260 /$ jemds $/ 2017 / 1231$ nerve examination was normal with no thickened or tender nerve appreciated. There was no sensory loss over hands and feet. Hands and feet did not show any wasting of muscles or deformities, callosities, fissures or ulcers. Mucosae were normal. Hair, nail and eye examinations were normal.

\section{CLINICAL DIAGNOSIS}

Patient was already a diagnosed case of Hansen's disease and was started on the treatment. But based on clinical features, we wanted to rule out post kala-azar dermal leishmaniasis (PKDL).

\section{INVESTIGATIONS}

CBC, LFT, RFT, chest x-ray, USG abdomen were all normal. His HIV1 and 2, VDRL tested negative. Slit skin smear for acid-fast bacilli was negative. Histopathology of punch biopsy from nodular lesion showed thin epidermis with follicular plugging at places, dense infiltrate all throughout the dermis composed of lymphocytes, histiocytes, plasma cells, neutrophils and macrophages with thin grenz zone seen. The macrophages showed basophilic oval dots suggestive of Leishman-Donovan bodies.

\section{FINAL DIAGNOSIS}

Considering the clinical features and confirmative histopathological features, patient was diagnosed as Post Kala-Azar Dermal Leishmaniasis.

\section{MANAGEMENT}

He was started on Miltefosine $50 \mathrm{mg}$ twice daily.

\section{DISCUSSION}

First described by Brahmachari in 1922, Post Kala-Azar Dermal Leishmaniasis (PKDL) is a dermal complication caused as a sequel to Visceral Leishmaniasis (VL). ${ }^{1}$ It has also been reported in individuals without prior history of VL as well as those undergoing treatment for VL. ${ }^{2}$

In India, it manifests in 5 - 15 percent of VL cases after months or several years of remission from infection. ${ }^{3}$ It is important to diagnose PKDL, as these patients are the reservoirs to transmit infection. There is increasing evidence that the pathogenesis is largely immunologically mediated; high concentrations of interleukin 10 in the peripheral blood of $\mathrm{VL}$ patients predict the development of PKDL. ${ }^{4} \mathrm{VL}$ is characterised by an absent immune response to Leishmania with a predominantly Th2 type of response with high levels of IL-10; after successful treatment, the patient will be immune with in vitro features of a Th1 type of response and in vivo a positive leishmanin skin test. PKDL takes an intermediate position with a dissociation of the immune response between the skin and the viscera with a Th2 and Th1 type of response, respectively. ${ }^{5}$ 
Clinically, the morphology and distribution of the lesions closely simulate that of leprosy, creating confusion in differentiating it from the latter. ${ }^{6}$ Papules, nodules, hypopigmented patches are the most common presentations of PKDL, which are often confused with Hansen's. Some patients with PKDL also develop neuritis of peripheral cutaneous nerves. ${ }^{7}$ Due to the close resemblance with Hansen's lesions, our patient was also initially diagnosed as Hansen's and started on multibacillary multidrug therapy. Although, rapid dipstick diagnostic test RK39 based on recombinant K39 protein shows a robust sensitivity of $96 \%$ to $100 \%$ and specificity of $97 \%$ to $100 \%$ in the studies conducted, ${ }^{8}$ it was negative in our patient. Demonstration of the parasites in slit skin smear, histopathology, tissue aspirates is the gold standard for diagnosis of PKDL. Conventional diagnosis using histopathology in PKDL tissue sections using haematoxylin and eosin ( $\mathrm{H}$ and $\mathrm{E})$ staining shows a variable degree of positivity for LD bodies ranging from $67-100$ percent in nodular lesions, $36-69$ percent in papular lesions and 7 - 33 percent in macular lesions. ${ }^{9,10}$

Thus, it is important to differentiate PKDL from Hansen's disease and thoroughly investigate a suspected case of PKDL in spite of negative RK39 test.

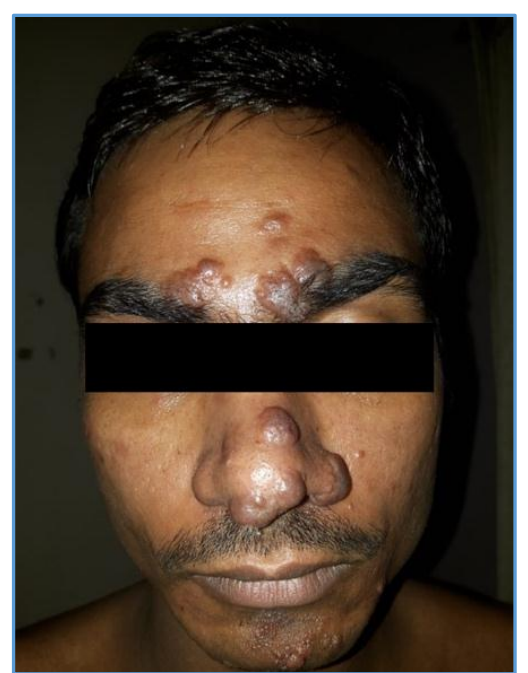

Figure 1

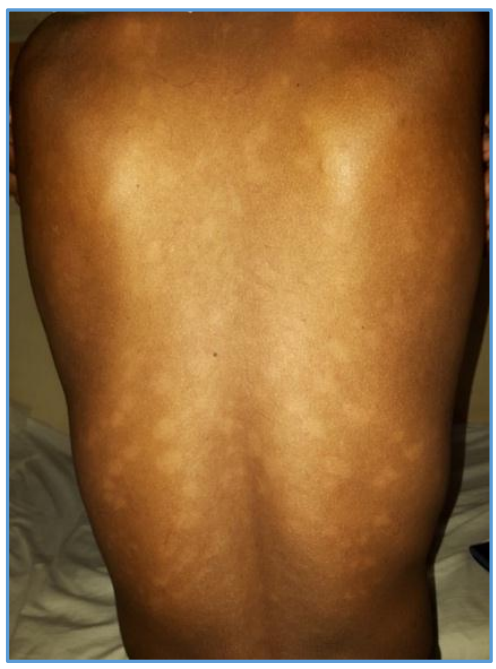

Figure 2

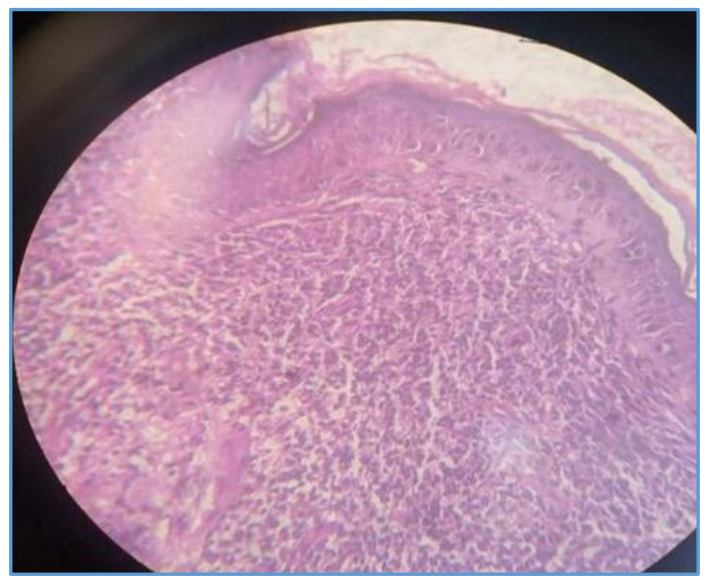

Figure 3

\section{REFERENCES}

[1] Salotra P, Singh R. Challenges in the diagnosis of post kala-azar dermal leishmaniasis. Indian J Med Res 2006;123(3):295-310.

[2] Adams ER, Versteeg I, Leeflang MMG. Systematic review into diagnostics for Post-Kala-Azar Dermal Leishmaniasis (PKDL). Journal of Tropical Medicine Article ID 150746, 2013;2013: p. 8.

[3] Arora S, D'Souza P, Haroon MA, et al. Post-kala-azar dermal leishmaniasis mimicking leprosy relapse: a diagnostic dilemma. Int J Dermatol 2014;53(5):606-8.

[4] Zijlstra EE, Musa AM, Khalil EA, et al. Post-kala-azar dermal leishmaniasis. Lancet Infect Dis 2003;3(2):8798.

[5] Zijlstra EE. The immunology of post-kala-azar dermal leishmaniasis (PKDL). Parasites \& Vectors 2016;9(1):464.

[6] Dhar S, Malakar S. Hypersensitivity reaction over lesions of Post-Kala-Azar dermal leishmaniasis mimicking type 1 reaction in leprosy. International Journal of Leprosy and Other Mycobacterial Diseases 1999;67(1):66-7.

[7] Elhassan AM, Ali MS, Zijlstra E, et al. Post-kala-azar dermal leishmaniasis in the Sudan: peripheral neural involvement. Int J Dermatol 1992;31(6):400-3.

[8] Das ML, Deb M, Karki BM, et al. Use of rK39 for diagnosis of post kala-azar dermal leishmaniasis in Nepal. Southeast Asian J Trop Med Public Health 2007;38(4):619-25.

[9] Ramesh V, Mukherjee A. Post-kala-azar dermal leishmaniasis. Int J Dermatol 1995;34(2):85-91.

[10] Sharma MC, Gupta AK, Verma N, et al. Demonstration of Leishmania parasites in skin lesions of Indian post kala-azar dermal leishmaniasis (PKDL) cases. J Commun Dis 2000;32(1):67-8. 